\title{
Bilateral Adrenocortical Carcinoma Presenting as Acute Adrenal Insufficiency
}

\author{
Anshita Aggarwal, Aprajita Verma, Aparajita Roy and Bindu Kulshreshtha \\ Department of Endocrinology, Atal Bihari Vajpayee Institute of Medical Sciences and Dr Ram Manohar Lohia Hospital, New Delhi, India
}

DOI: https://doi.org/10.17925/EE.2020.16.2.172

$\mathrm{T}$ he presentation of adrenocortical carcinoma (ACC) with bilateral adrenal masses and acute adrenal insufficiency is exceedingly rare and has only been seen in anecdotal case reports. Herein, we describe the case of a 50-year-old male who presented with a painful abdominal lump, loss of weight and appetite, several episodes of vomiting, and hypotension. Imaging studies were suggestive of bilateral adrenal masses and a lung mass. Hormonal work-up was suggestive of acute adrenal insufficiency, with the adrenal masses being non-secretory. An ultrasound-guided biopsy from one of the adrenal masses was suggestive of ACC, thus confirming the diagnosis as bilateral ACC with distant metastases. Bilateral adrenalectomy was planned for the patient, but unfortunately, he succumbed to his disease before the surgery.

\section{Keywords}

Adrenocortical carcinoma, bilateral adrenal masses, acute adrenal insufficiency

Disclosures: Anshita Aggarwal, Aprajita Verma, Aparajita Roy and Bindu Kulshreshtha have no financial or non-financial relationships or activities to declare in relation to this article. Review Process: Double-blind peer review.

Compliance with Ethics: Informed consent was received from the patient involved in this case study; no identifying information or images have been included.

Authorship: The named authors meet the International Committee of Medical Journal Editors (ICMJE) criteria for authorship of this manuscript, take responsibility for the integrity of the work as a whole, and have given final approval for the version to be published.

Access: This article is freely accessible at

touchENDOCRINOLOGY.com

(C) Touch Medical Media 2020

Received: 14 June 2020

Accepted: 27 July 2020

Published Online: 30 September 2020

Citation: European Endocrinology. 2020;16(2):172-4

Corresponding Author: Anshita Aggarwal,

Department of Endocrinology, ABVIMS and Dr RMLH, Baba Kharak Singh Marg, Guest House, Room No. 306, New Delhi 110001 India. E: anshilh10@gmail.com

Support: No funding was received for

the publication of this article.
Adrenocortical carcinoma (ACC) is a rare malignancy, and presentation as bilateral adrenal masses and acute adrenal insufficiency is even rarer. Due to the relative commonality of other causes for bilateral adrenal masses, such as metastases or infiltrative diseases, they take precedence while considering differential diagnoses. We present the case of a gentleman who presented with bilateral adrenal masses due to ACC with distant metastases, and also acute adrenal insufficiency. The case posed a diagnostic dilemma owing to the rare presentation. This case has a relevance in the clinical context as the management of other causes of bilateral adrenal masses is completely different to that of ACC, and hence it is imperative not to miss the diagnosis.

\section{Case report}

A 50-year-old male presented to us complaining of dull-aching, left-sided abdominal pain with a lump at the same site, which he had noticed over the last 6 months. He also gave a history of occasional episodes of vomiting associated with poor appetite and a significant loss of weight. He did not give any history of change in bowel or bladder habits, jaundice, or fever. He had never been hospitalised previously and did not report any chronic medical conditions. He was a chronic smoker (60 pack-years) and did not have any other addictions.

On examination, the patient had a lean build, with a large, irregular abdominal mass palpable in the left lumbar and iliac regions, which was hard in consistency. He had hypotension (blood pressure $90 / 60 \mathrm{mmHg}$ ), and did not have any stigmata of Cushing's syndrome. Routine investigations revealed iron-deficiency anaemia, hypoproteinaemia (total serum protein and albumin were $6.3 \mathrm{mg} / \mathrm{dL}$ and $2.8 \mathrm{mg} / \mathrm{dL}$, respectively), hyponatraemia (serum sodium $124 \mathrm{mEq} / \mathrm{L}$ ), hyperkalaemia (serum potassium $6.2 \mathrm{mEq} / \mathrm{L}$ ), and transaminitis (aspartate aminotransferase and alanine aminotransferase: 1,030 and $23 \mathrm{IU} / \mathrm{L}$, respectively). An ultrasound of the abdomen showed bilateral suprarenal masses, and contrast-enhanced computerised tomography (CT) was then carried out for further characterisation of the masses. It revealed ill-defined, heterogeneous, mildly enhancing, solid, soft-tissue attenuation masses in bilateral suprarenal location $(17.0 \times 19.8 \times 13.2 \mathrm{~cm})$; bilateral adrenal glands were not visible separately (Figure 1). The mass was invading the right lobe of the liver and the intrahepatic inferior vena cava. It was also displacing the stomach, pancreas and spleen, with loss of fat planes, and was causing encasement of the splenic vein, aorta, celiac axis and bilateral main renal arteries. Multiple necrotic, retroperitoneal lymph nodes in aortocaval, pre-aortic and left para-aortic locations were also seen. A large, irregular, soft-tissue attenuation mass $(4 \mathrm{~cm})$ was seen within the superior segment of the left lower lobe of the lung (Figure 2), with smaller nodules in the right upper and middle lobes. There was also an anterior wedge collapse of the L2 vertebral body.

A possibility of malignancy with disseminated metastases, including bilateral adrenal metastases, was considered. Owing to the rarity of bilateral ACC, and keeping the possibility of bilateral adrenal metastases in mind, an ultrasound-guided biopsy was carried out from the left-sided adrenal mass, before which, a complete endocrine work-up was performed. Basal cortisol (8 am) was $331 \mathrm{nmol} / \mathrm{L}$, 
Figure 1: Contrast-enhanced computed tomography of the abdomen showing bilateral solid, heterogenous, ill-defined adrenal masses

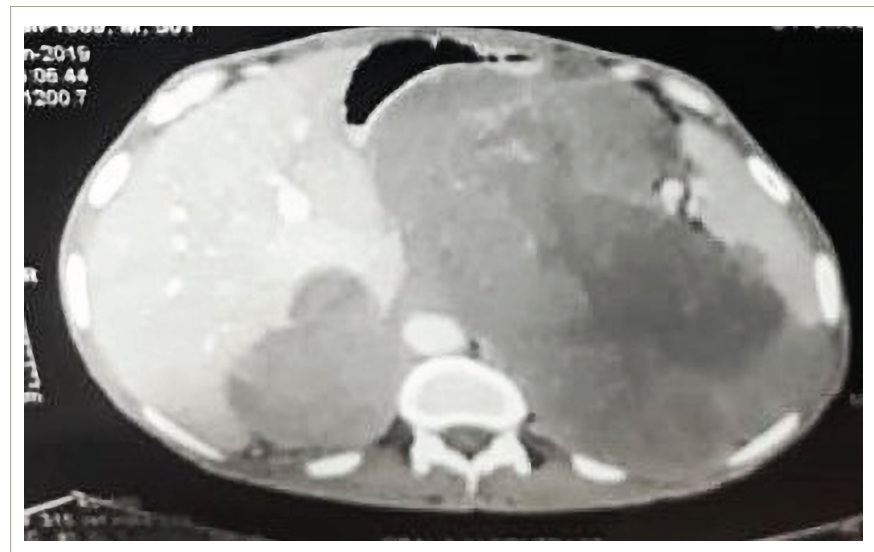

Figure 2: Contrast-enhanced computed tomography of the chest showing a large, irregular mass in the left lower lobe of the lung

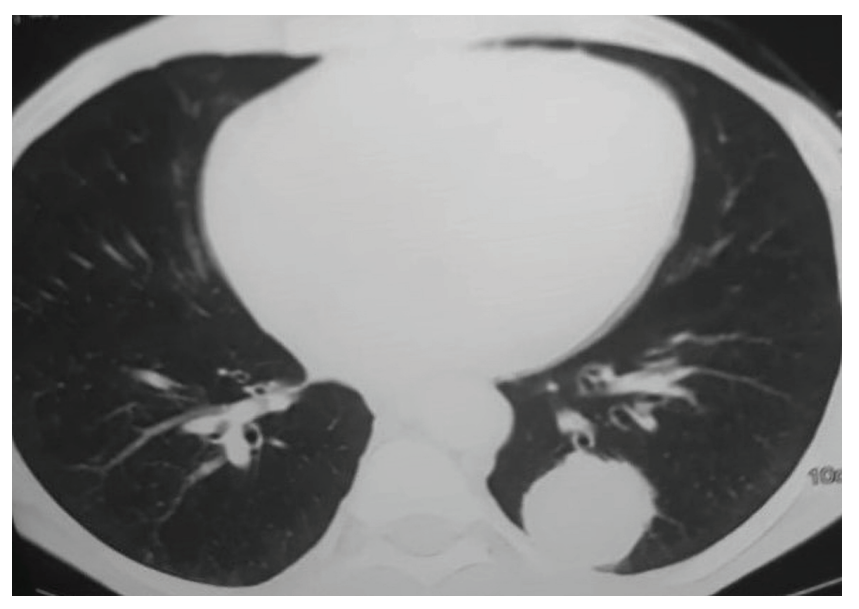

and after adrenocorticotropic hormone (ACTH) stimulation with $250 \mu \mathrm{g}$ synacthen, the peak cortisol value was $509 \mathrm{nmol} / \mathrm{L}$ (increment of $178 \mathrm{nmol} / \mathrm{L}$ ), suggestive of relative adrenal insufficiency in the setting of a critical illness. ${ }^{1}$ Dehydroepiandrosterone sulfate level was $1.2 \mu \mathrm{g} / \mathrm{L}$ (normal range: $0.4-4.6 \mu \mathrm{g} / \mathrm{L}$ ). The rest of the hormonal work-up (thyroid function test, gonadotropins, prolactin) was normal. Histopathological findings were suggestive of ACC, and the immunohistochemistry was also positive for adrenal cortical specific markers (calretinin, inhibin and melan A), and was negative for chromogranin (Figures 3 and 4). The patient was initiated on glucocorticoid and mineralocorticoid replacement, following which, he had a transient improvement in blood pressure and was scheduled for bilateral adrenalectomy. However, he succumbed to his illness before he could be taken up for surgery.

\section{Discussion}

We described a case of adrenal insufficiency due to bilateral adrenal masses, which on evaluation was found to be due to ACC. Primary malignant tumours originating from the adrenal gland include ACCs, primary adrenal lymphomas and malignant pheochromocytomas. The common differential diagnoses for bilateral adrenal masses
Figure 3: Haematoxylin and eosin stain (20x) adrenocortical carcinoma with marked nuclear polymorphism, hyperchromasia, bi- and multi-nucleation

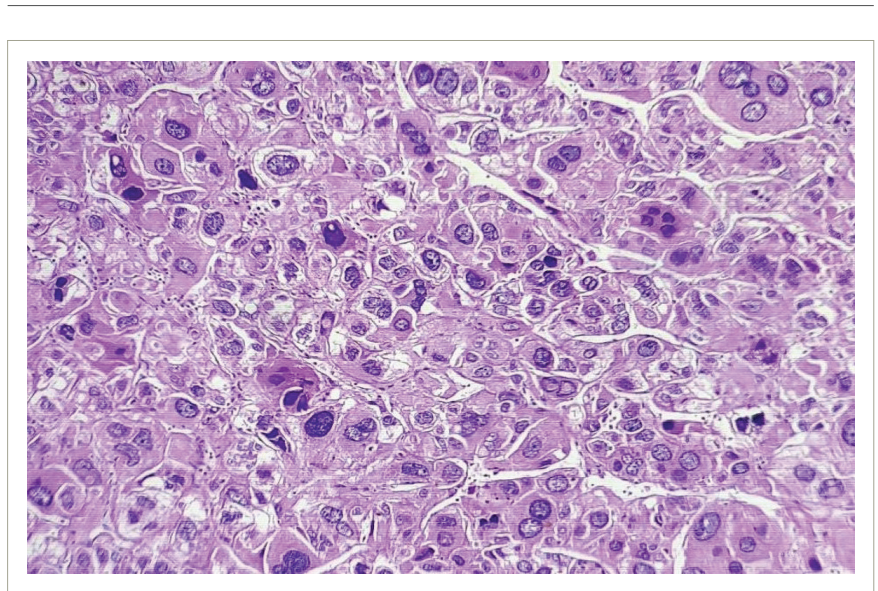

include infective aetiologies, such as tuberculosis and histoplasmosis; infiltrative disorders, such amyloidosis, metastasis from an unknown primary, non-Hodgkin's Iymphoma; bilateral pheochromocytoma; and congenital adrenal hyperplasia., The presentation of ACC as bilateral adrenal masses is quite uncommon (seen in $2-10 \%$ of cases of ACC), especially because ACC itself is a rare neoplasm with an annual incidence of $0.7-2.0$ cases/million. ${ }^{4}$ It has a bimodal age distribution, with one peak in children younger than 5 years and another peak between the ages of 30 and 50 years. ${ }^{5}$ Forty to sixty percent of ACCs are secretory, while the rest are non-secretory. ${ }^{6}$ The presentation of bilateral ACC as adrenal insufficiency is even rarer. To the best of our knowledge, this is the third case ever reported of bilateral ACC with adrenal insufficiency. ${ }^{7.8}$ In one of the previously reported cases, bilateral ACC co-existed with pulmonary tuberculosis, which itself is known to cause adrenal insufficiency.

The diagnosis of acute adrenal insufficiency in critically ill patients, as in the index case, deserves special mention as it has always remained a matter of debate. Though consensus is lacking, a recent review has shown that in the background of hypotension, suggesting critical illness with a serum albumin of $>2.5 \mathrm{mg} / \mathrm{dL}$, a baseline serum cortisol level $<400 \mathrm{nmol} / \mathrm{L}(15 \mathrm{\mu g} / \mathrm{dL})$ itself suggests cortisol insufficiency, as was seen in the index case. ${ }^{9}$ Studies have also shown that a delta rise (difference between stimulated and basal values) in cortisol after ACTH stimulation test of $<9 \mu \mathrm{g} / \mathrm{dL}(<250 \mathrm{nmol} / \mathrm{L})$ in critically ill patients is a poor prognostic marker and is associated with increased mortality. ${ }^{1}$ Further, there is also controversy regarding the 1 versus $250 \mu$ g synacthen dose used in ACTH stimulation tests to detect adrenal insufficiency in critically ill patients. ${ }^{1}$ As most critical care studies have reported the use of a $250 \mu \mathrm{g}$ dose, it is advisable to use the same. ${ }^{9}$

There is no evidence to suggest whether the contralateral gland is involved in cases of bilateral ACC, or is involved due to metastases from the other gland. The rarity of bilateral ACC, coupled with the fact that bilateral adrenal masses in sick patients are more commonly due to metastases or infections, can mean that bilateral ACC may be missed. The presence of a known primary malignancy almost always means that bilateral adrenal masses are metastatic in origin. However, in the absence of a known primary, clinical clues may not offer much help. The radiological features of ACC and metastases are also very similar, with the radiodensity on unenhanced $\mathrm{CT}$ being 


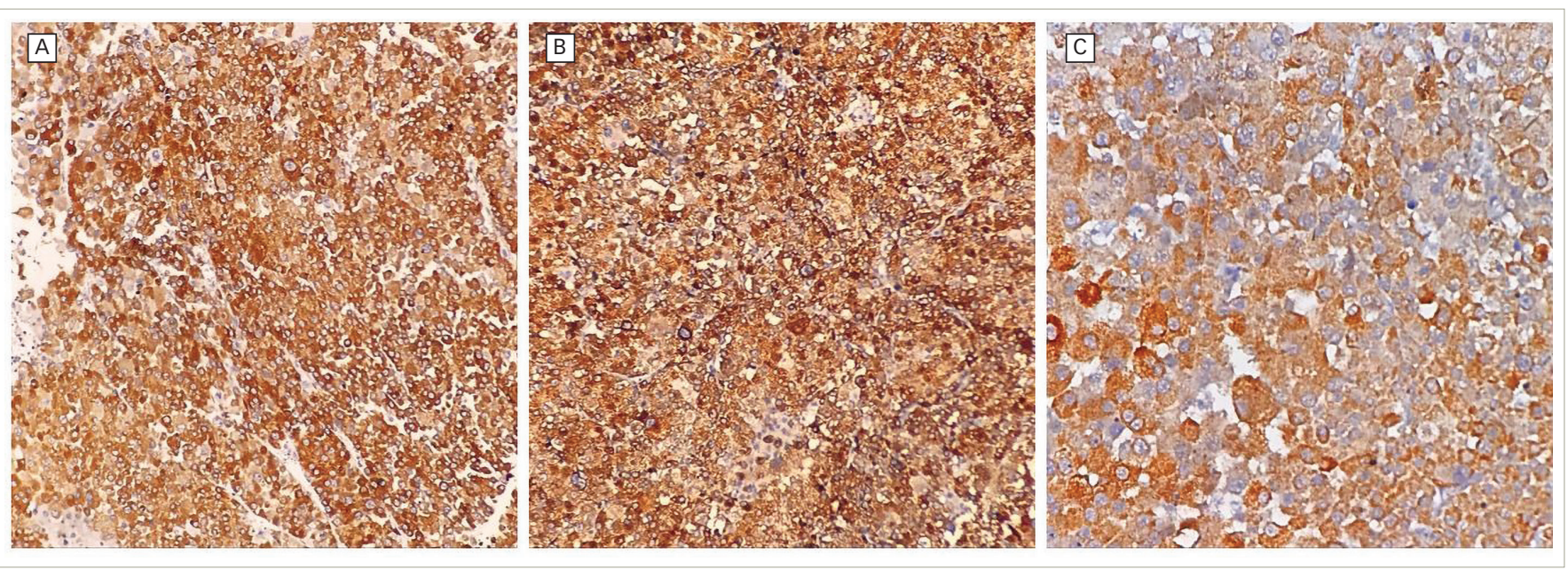

$>10$ Hounsfield units with a post-contrast washout of $<50 \%$, in both cases. An ${ }^{18} \mathrm{~F}$-fluorodeoxyglucose positron emission tomography scan cannot differentiate adrenal metastases from ACC, except if there is significant uptake (as seen by the standardised uptake value max) in the primary extra-adrenal lesion, in the case of adrenal metastases. CT-guided fine-needle aspiration is recommended in the case of suspected adrenal metastases, while on the contrary, it is to be avoided in ACC, as it increases the risk of widespread dissemination.

In the index patient, because of the bilateral nature of the disease, the degree of suspicion for ACC was low, hence we proceeded with adrenal biopsy. However, the histopathological findings were suggestive of ACC. Genetic analysis could not be done. A previous case report of a patient with bilateral ACC demonstrated allelic losses of the p53 and RB genes in both adrenal tumours. ${ }^{10}$ The treatment of ACC versus metastatic adrenal masses is diametrically different. While metastatic disease necessitates treatment for the underlying primary malignancy, the treatment of bilateral ACC encompasses bilateral adrenal gland removal with lifelong glucocorticoid and mineralocorticoid replacement. The further course of treatment is dependent on the European Network for the Study of Adrenal Tumor (ENSAT) staging of the tumour. ${ }^{11}$

To conclude, our case represents a rare presentation of ACC and we suggest that ACC should be considered in the differential diagnoses of bilateral adrenal masses with acute adrenal insufficiency. •
1. Annane D, Sébille V, Troché G, et al. A 3-level prognostic classification in septic shock based on cortisol levels and cortisol response to corticotropin. JAMA. 2000;283:1038-45.

2. Mukherjee JJ, Villa ML, Tan L, et al. Bilateral adrenal masses due to histoplasmosis. J Clin Endocrinol Metab. 2005:90:6725-6.

3. Kita M, Mandala E, Saratzis A, et al. Primary adrenal lymphoma presenting as Addison's disease. Case report and review of the literature. Exp Clin Endocrinol Diabetes. 2008;116:363-5.

4. Wooten MD, King DK. Adrenal cortical carcinoma. Epidemiology and treatment with mitotane and a review of the literature. Cancer. 1993;72:3145-55.
5. Wasserman JD, Zambetti GP, Malkin D. Towards an understanding of the role of p53 in adrenocortical carcinogenesis. Mol Cell Endocrinol. 2012;351:101-10 6. Venkatesh S, Hickey RC, Sellin RV, et al. Adrenal cortical carcinoma. Cancer. 1989:64:765-9.

Chattopadhyay B, Bhattacharya B, Chatterjee A, Debnath NB. Bilateral adrenocortical carcinoma presenting as acute adrena insufficiency with co-existing active pulmonary tuberculosis: A diagnostic dilemma. Clin Cancer Investig J. 2013;2:371-3.

8. Kim MJ, Kim JH, Kim TY, Kim SW. A case report of bilatera adrenocortical carcinoma complicated by adrenal insufficiency. Endocrinol Metab. 2011;26:243-7.
9. Hamrahian AH, Fleseriu M; AACE Adrenal Scientific Committee. Evaluation and management of adrenal insufficiency in critically ill patients: disease state review. Endocr Pract. 2017;23:716-25.

10. Miyamoto $\mathrm{H}$, Kubota Y, Shuin T, Shiozaki H. Bilateral adrenocortical carcinoma showing loss of heterozygosity at the p53 and RB gene loci. Cancer Genet Cytogenet. 1996:88:181-3.

11. Fassnacht M, Dekkers OM, Else T, et al. European Society of Endocrinology Clinical Practice Guidelines on the management of adrenocortical carcinoma in adults, in collaboration with the European Network for the Study of Adrenal Tumors. Eur J Endocrinol. 2018;179:G1-46. 